\title{
The Giant Magellan Telescope Laser Tomography Adaptive Optics System
}

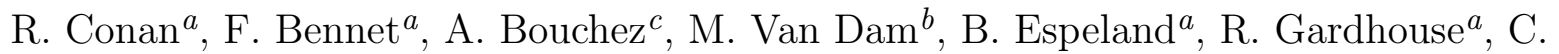 \\ d'Orgeville $^{a}$, S. Parcell ${ }^{a}$, P. Piatrou ${ }^{a}$, I. Price ${ }^{a}$, F. Rigaut ${ }^{a}$, G. Trancho ${ }^{c}$ and K. Uhlendorf ${ }^{a}$ \\ ${ }^{a}$ RSAA, The Australian National University, Weston Creek, ACT 2611, Australia \\ ${ }^{b}$ Flat Wavefronts, P.O. Box 1060, Christchurch 8140, New-Zealand \\ ${ }^{c}$ GMTO, P.O. Box 90933, Pasadena, CA 91109, USA
}

\begin{abstract}
The Giant Magellan Telescope presents a unique optical design with seven $8.4 \mathrm{~m}$ diameter primary mirrors matched by seven adaptive secondary mirrors (ASM). The ASMs can be controlled in several different Adaptive optics (AO) observing modes coupled to the telescope. One of these AO systems, the Laser Tomography Adaptive Optics (LTAO) system is currently in its preliminary design phase. The LTAO observing mode will provide a Strehl ratio in $\mathrm{H}$ band of at least $30 \%$ over more than $20 \%$ of the sky and an ensquared energy in $\mathrm{K}$ band of at least $40 \%$ in a 50 milli-arcsec spaxel over more than $50 \%$ of the sky. To achieve its performance requirements, the LTAO observing mode uses six 20W Laser Guide Stars (LGS) with six order-50x50 ShackHartmann wavefront sensors. The LGSs are launched from three locations at the periphery of the telescope primaries. A natural guide star (NGS) is used separately to measure tip-tilt, focus and low-bandwidth-loworder aberrations. A open-loop controlled deformable mirror corrects the off-axis NGS infrared wavefront. We give an update on the design of the LTAO WFSs, the LGS facility, the on-instrument wavefront sensors and the tomography and control algorithms.
\end{abstract}

\section{INTRODUCTION}

The Giant Magellan Telescope ${ }^{5}$ (GMT) is one the next generation extremely larger optical telescope. The GMT optical design includes an Adaptive Secondary Mirror (ASM) to allow the scientific instruments to benefit of the advantages of Adaptive Optics ( $\mathrm{AO}$ ) while maintaining the telescope high throughput.

The primary goal of adaptive optics on the GMT ${ }^{3}$ is the correction of atmospheric blurring to recover images with the diffraction-limited beam profile, thus providing the GMT with correspondingly improved sensitivity and resolution. The GMT large aperture holds the potential of uniquely sharp images, three times sharper than the limit for an $8 \mathrm{~m}$ aperture. The promise of an extremely large telescope (ELT) with LTAO, combining sharper resolution at the diffraction limit with a huge collecting area, has quite clear scientific benefit. ${ }^{8}$

The GMT has a Gregorian optical design, with an f/0.7 primary mirror composed of $78.4 \mathrm{~m}$ diameter circular segments and an identically segmented concave secondary mirror. The area of the primary mirror is equivalent to that of a $21.9 \mathrm{~m}$ filled circular aperture with the same central obscuration. The optics are actively supported in a compact alt-azimuth steel telescope structure which, including its $12 \mathrm{~m}$ high concrete pier, has a mass of 1,125 metric tons and a lowest resonant frequency of $4.5 \mathrm{~Hz}$. A large enclosure surrounds the telescope and protects it from wind disturbance and inclement weather.

The GMT AO system design is based on an adaptive secondary mirror (ASM), and wavefront sensor assemblies replicated for each instrument. All narrow-field AO instruments are located at a folded Gregorian focus provided by a steerable tertiary mirror. Each instrument cryostat window is a long-pass dichroic, and all visible-light wavefront sensing is performed in the beam reflected by the window. This design provides very high throughput and low emissivity, with only 3 optical surfaces between the sky and the science instrument. Fig. 1 shows a block diagram of the LTAO and Natural Guide Star AO (NGSAO) observing modes.

\footnotetext{
Send correspondence: rconan@mso.anu.edu.au
} 


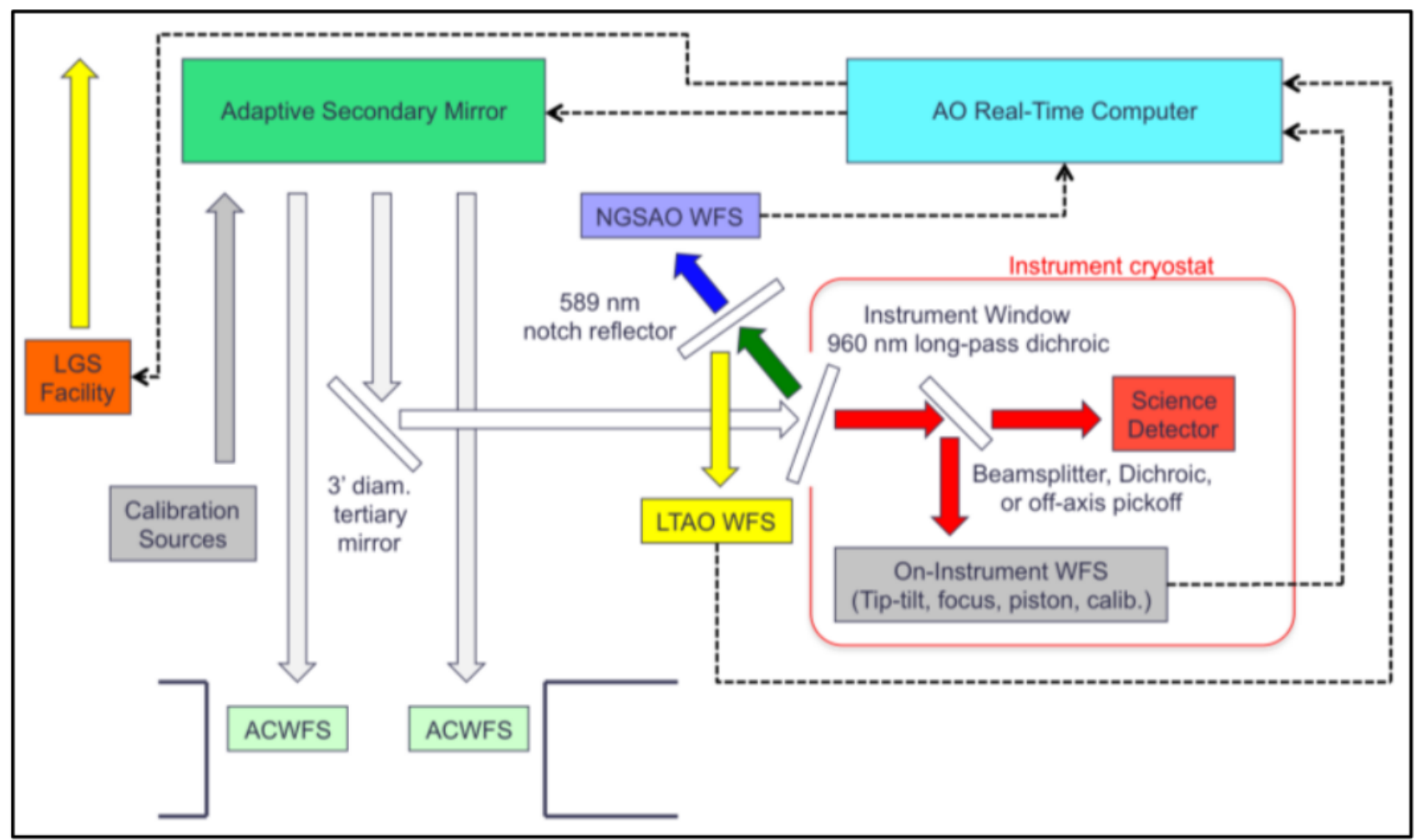

Figure 1. Block diagram of the NGSAO and LTAO modes. Light paths are shown with wide arrows, control signals with dashed arrows.

The Ground Layer $\mathrm{AO}^{4}$ (GLAO) observing mode is only used by wide-field instruments which can be translated to the direct Gregorian focus. The AO system therefore provides a single GLAO wavefront sensing assembly, intercepting laser guide stars and natural guide stars with a large dichroic and pick- off mirrors ahead of the instrument entrance window.

Laser Tomography adaptive optics (LTAO) uses a 60" diameter constellation of 6 LGS to tomographically reconstruct the high-order components of the atmospheric wavefront aberrations in the direction of a central science target. One faint natural guide star is used to measure tip-tilt, truth, focus and segment piston terms in the infrared. The wavefront aberration will be compensated by the ASM, providing diffraction-limited imaging at wavelengths longer than $1 \mu \mathrm{m}$ over a field of view limited by atmospheric isoplanatism. Sky coverage is greatly increased over the Natural Guide Star AO (NGSAO) observing mode, with the design presented herein achieving greater than $50 \%$ sky coverage over the entire sky.

In LTAO Mode, the AO System uses one NGS to provide data for Tip-Tilt, Truth, Focus and Segment Piston. Tip-tilt uses infrared light in the $\mathrm{K}$ band. Focus, Truth \& Piston are sensed using light from J band. This operational concept assumes a combined On Instrument WFS for Tip-Tilt, Focus, Truth and Piston such that the Tip-Tilt sensor will be physically aligned with the wavefront sensor for Focus, Truth \& Piston. The acquisition FoV for the NGS is shown in Fig. 2. Also shown in Fig. 2 is the LGS constellation with 6 laser guide stars equally spaced on a 60 " diameter circle around the optical axis. A nominal science FoV is also shown. The LTAO Mode also requires the Active Optics System and Segment Piston Subsystem to be functioning.

\section{REQUIREMENTS}

The LTAO design is constrained by the following Science Requirements. 


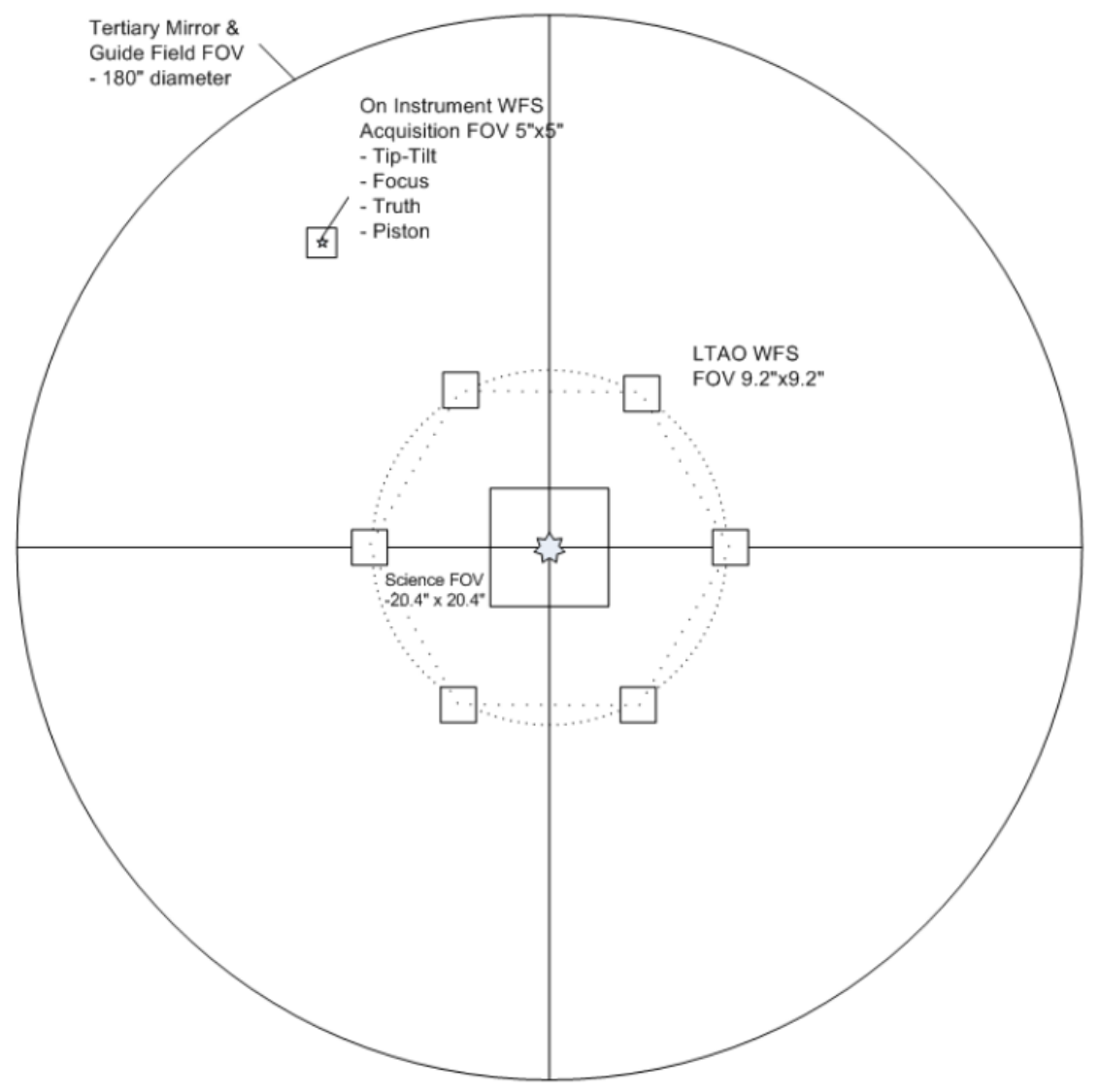

Figure 2. LTAO Guide Field Configuration Example

\subsection{Science Requirements}

LTAO Capability The GMT shall provide a LTAO observing mode to achieve diffraction- limited image quality 0.96-14 m wavelength (goal: $0.90-25 \mathrm{~m}$ ) over a large fraction of the observable sky, with a field of view limited by atmospheric anisoplanatism.

LTAO Imaging with Moderate Sky Coverage The GMT in LTAO observing mode shall deliver an on-axis $\mathrm{H}$ band $(1.65 \mathrm{~m})$ Strehl ratio of no less than 0.30 over at least $20 \%$ of the sky at the galactic pole, over a 120 s integration (goal: $50 \%$ at the galactic pole).

LTAO Spectroscopy with High Sky Coverage The GMT in LTAO observing mode shall deliver a K band $(2.2 \mathrm{~m})$ fractional ensquared energy in 50x50 mas of no less than 0.40 over at least $50 \%$ of the sky at the galactic pole, over a $900 \mathrm{~s}$ integration (goal: $90 \%$ of the sky at the galactic pole).

LTAO Spectroscopy with On-Axis Infrared Guide Star The GMT in LTAO observing mode shall deliver a $\mathrm{K}$ band $(2.2 \mathrm{~m})$ fractional ensquared energy in $85 \times 85$ mas of no less than 0.5 , and a PSF FWHM no greater than 20 . mas, when using an on-axis $\mathrm{K}=15$ guide star, over a $900 \mathrm{~s}$ integration.

\subsection{LTAO Mode Performance Requirements}

From the Science Requirements are derived the LTAO Mode Performance Requirements: ${ }^{12}$

LTAO High-Order Error with Moderate Coverage The AO system in LTAO mode shall have a high order error less than 230 . nm RMS over $20 \%$ of the sky at the galactic pole, over a 120 s integration [goal: $50 \%$ sky coverage]. 
LTAO Image Motions with Moderate Sky Coverage The AO system in LTAO mode shall have an LTAO image motion error less than 2. milliarcsec RMS over $20 \%$ of the sky at the galactic pole, over a $120 \mathrm{~s}$ integration [Goal: $50 \%$ sky coverage].

LTAO High-Order Error with High Sky Coverage The AO system in LTAO mode shall have an LTAO High Order error less than 250. nm RMS over $50 \%$ of the sky at the galactic pole, over a 1800 s integration.

LTAO Image Motions with High Sky Coverage The AO system in LTAO mode shall have an LTAO image motion error less than 5.5 milliarcsec RMS over $50 \%$ of the sky at the galactic pole, over a 1800 s integration.

LTAO High-Order Error with On-Axis Infrared Guidestar The AO system in LTAO mode shall have a high order error less than 205. nm RMS when using a $\mathrm{K}=15$ on-axis natural guidestar, over a $1800 \mathrm{~s}$ integration.

LTAO Telescope Segment Piston Error The AO system in LTAO mode shall have a Telescope Segment Piston error less than 50.

nm RMS over $50 \%$ of the sky at the galactic pole, over a $120 \mathrm{~s}$ integration [goal: $70 \mathrm{~nm}$ over $90 \%$ sky coverage].

LTAO Non-Common Path Aberration Stability The AO System in LTAO mode shall maintain non-common path errors less than 64 . nm throughout a 1800s integration.

\section{THE GMT PUPIL}

The GMT pupil is the results of the projection of the primary segments onto the secondary segments. It consists in 7 circular mirrors arranged as shown in Fig. 3. The main parameters of the GMT segmented pupil are given in Tab. 1.

The point-spread function (PSF) corresponding to the GMT pupil is plotted in Fig. 4 (middle picture). The GMT PSF can be interpreted as the product of the PSF of a $8 \mathrm{~m}$ diameter pupil (Fig. 4, left image) with the interference patterns of the GMT seven segments (Fig. 4, right image).

Table 1. GMT pupil parameters, units are meters

\begin{tabular}{cccc}
\hline$D$ & $D_{0}$ & $D_{h}$ & $b$ \\
\hline 8.365 & 3.2 & 0.55 & 8.658 \\
\hline
\end{tabular}

\section{LASER TOMOGRAPHY}

At the root of any LTAO system, there is the tomographic algorithm which estimate the turbulence wavefront in a given direction on the sky based on a set of measurements from different directions. The baseline algorithm for the GMT LTAO mode is a Linear Minimum Mean Square Error ${ }^{6}$ (LMMSE) reconstructor.

The formalism developed below is using the same notations as in Ref. ${ }^{2}$ The LMMSE reconstruction consists in the estimation of a DM actuator command vector $a$ that best compensates for the atmospheric turbulence wavefront $x_{0}$ of an on-axis NGS in the telescope pupil. The LMMSE reconstruction derives the command vector $a$ such as the variance $\sigma^{2}$ of the residual wavefront error $\phi$ is minimized. The main assumption of LMMSE reconstruction is that $x_{0}$ can be written as a linear sum of the WFS measurement vector $\mathrm{s}$. The LMMSE reconstruction can be described by the relations

$$
\begin{aligned}
x_{0} & =E s \\
\phi & =x_{0}-a \\
\sigma^{2} & =\left\langle\phi^{T} \phi\right\rangle
\end{aligned}
$$

The WFS measurement vector $s$ is related to the atmospheric turbulence wavefront $x$ of off-axis sources (NGS and/or LGS) in the telescope pupil,

$$
s=G x+n,
$$




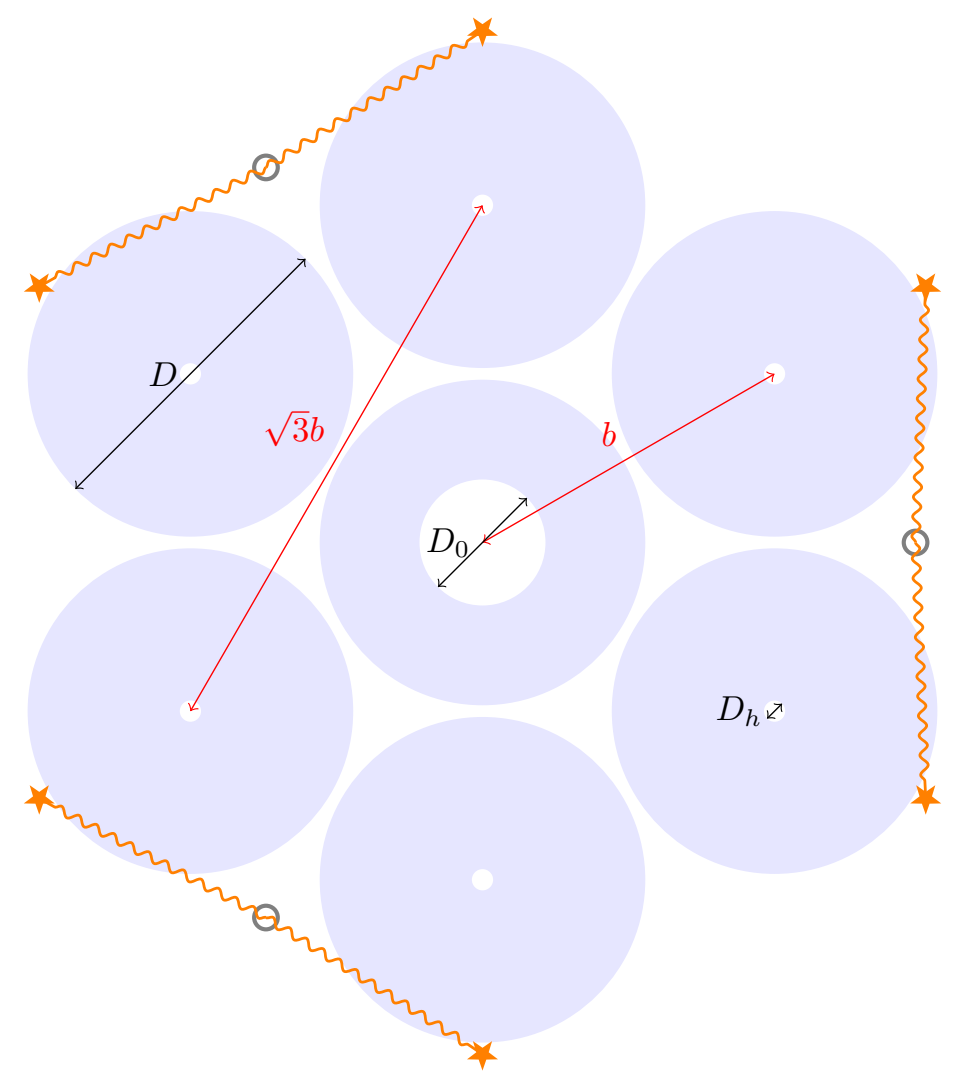

Figure 3. The GMT pupil with the location of the launch telescopes and Laser guide stars.

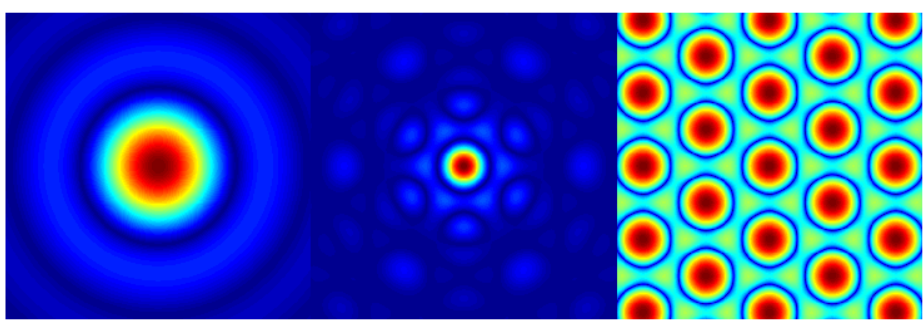

Figure 4. From left to right: $8 \mathrm{~m}$ diameter telescope PSF, GMT PSF and GMT segments interference pattern. The square root of the PSFs are plotted. 
where the matrix $G$ is the wavefront-to-WFS influence matrix and the vector $n$ is the WFS measurement noise. The objective of the LMMSE reconstruction is to derive the minimum variance reconstructor matrix $E$ that minimizes $\sigma^{2}$. The LMMSE reconstruction is re-written

$$
\begin{aligned}
\phi & =E(G x+n)-a \\
\sigma^{2} & =\left\langle\phi^{T} \phi\right\rangle
\end{aligned}
$$

and the minimization of $\sigma^{2}$ leads to ${ }^{6}$

$$
\begin{aligned}
E & =\left\langle a(G x+n)^{T}\right\rangle\left\langle(G x+n)(G x+n)^{T}\right\rangle^{-1} \\
& =\left\langle a x^{T}\right\rangle G^{T}\left[G\left\langle x x^{T}\right\rangle G^{T}+\left\langle n n^{T}\right\rangle\right]^{-1}
\end{aligned}
$$

The variance of the residual wavefront error is deduced from the covariance matrix of the residual wavefront error,

with

$$
\sigma^{2}=N_{a}^{-1} \operatorname{trace}\left\{\left\langle\phi \phi^{T}\right\rangle\right\}
$$

$$
\left\langle\phi \phi^{T}\right\rangle=\left\langle a a^{T}\right\rangle-\left\langle a x^{T}\right\rangle G^{T}\left[G\left\langle x x^{T}\right\rangle G^{T}+\left\langle n n^{T}\right\rangle\right]^{-1} G\left\langle x a^{T}\right\rangle
$$

where $N_{a}$ is the total number of actuators. Eq. (7) and Eq. (10) involves 5 matrices:

$G$ the wavefront-to-WFS influence matrix,

$\left\langle x x^{T}\right\rangle$ the covariance matrix of the wavefronts between the different off-axis sources,

$\left\langle a x^{T}\right\rangle$ the covariance matrix between the on-axis NGS wavefront and the wavefronts of the different off-axis sources,

$\left\langle a a^{T}\right\rangle$ the covariance matrix of the on-axis NGS wavefront,

$\left\langle n n^{T}\right\rangle$ the WFS measurement noise covariance matrix.

In the case of the GMT with 6 LGSs, $\left\langle x x^{T}\right\rangle$ and $\left\langle a x^{T}\right\rangle$ are $6 \times 6$ block and $1 \times 6$ block matrices, respectively. One block is the spatio-angular covariance at the telescope pupil between two wavefronts from two sources. The wavefront-to-WFS influence matrix is given by $G=D F^{\dagger}$ where $D$ is the poke matrix and $F$ is the ASM influence function matrix.

The ASM command vector $a$ is derived from $a=E s$ with $\mathrm{E}$ given by Eq. (7). The covariance matrices in the expression of the tomographic reconstructor $E$ are derived from the open-loop statistics of the optical turbulence meaning that the measurements vector $s$ should corresponds to uncorrected turbulence aberrations. The LGS WFSs are located after the ASM, $s$ is then a vector of partially corrected turbulence aberrations. In this closed-loop system, it is necessary to estimate the open-loop measurements from both the DM commands and the closed-loop measurements. The closed-loop tomography control algorithm uses the LGS wavefront sensor measurements vector $s_{n}$ and the last DM command vector $c_{n-1}$ to derive the new DM command vector $c_{n}$. It can be decomposed in the following steps

1. $\hat{s}_{n-1}^{D M}=D c_{n-1}$, estimation of the last LGS WFS slopes corresponding to last DM command using the DM to WFS interaction matrix $D$,

2. $s_{n}^{o l}=s_{n}-\hat{s}_{n-1}$, computing of the open-loop LGS WFS measurements,

3. $\hat{c}_{n}=E s_{n}^{o l}$, estimation of the DM command vector using the tomographic reconstructor $E$,

4. $c_{n}^{\varepsilon}=\hat{c}_{n}+c_{n-1}$, computing of the DM command residue i.e. the difference between the estimates of the next and of the previous DM commands,

5. $c_{n}=c_{n-1}-g c_{n}^{\varepsilon}$, integration of the DM command residue with a gain $g$. 

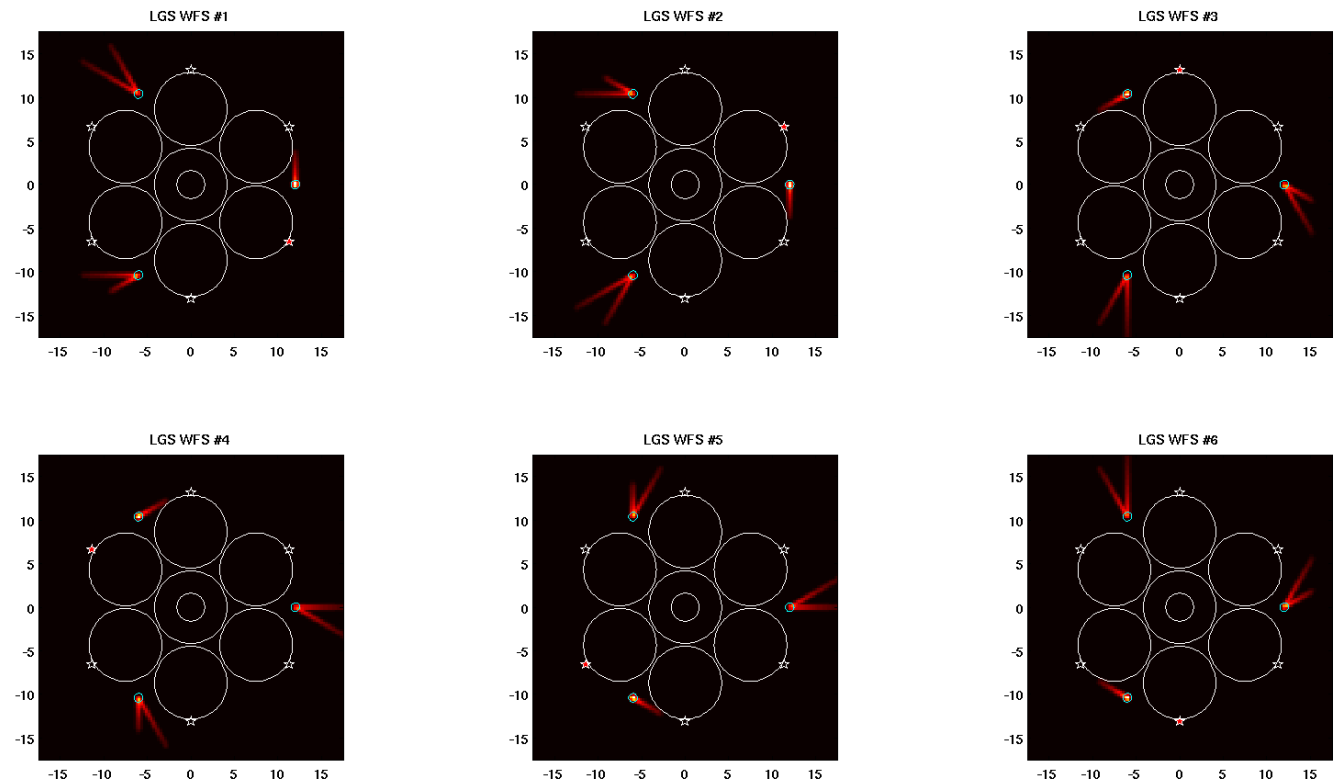

Figure 5. The fratricide effects as seen by the 6 LGS WFSs in the case of launching the Lasers from 3 locations at the telescope edge.

\section{LASER GUIDE STAR FACILITY}

The Laser Guide Star Facility (LGSF) of the GMT is using 6 LGSs evenly located on the rim of a circle of 60" diameter centered on the telescope axis. The LGSs are launched from the edge of the primary mirror at 3 locations (Fig. 3 and Fig. 7), 11.5m away from the telescope optical axis. Each location will host 2 launch telescopes.

This configuration minimizes fratricide effects (Fig. 5) while not impacting the performance of the on-axis tomographic reconstruction. Fig. 6 shows 3 tomographic wavefront error maps. From left to right, the maps correspond to 3 different launch telescope locations, within the primary segments, from the telescope edge and behind the ASM. The WFE maps are plotted as a function of the LGS WFS frame rate and as a function of the Laser power. The launch telescope locations have no significant impact on the WFE. The maps show that $120 \mathrm{~nm}$ rms WFE is achievable with a $20 \mathrm{~W}$ Laser and a frame rate of $500 \mathrm{~Hz}$.

\section{LASER GUIDE STAR WAVEFRONT SENSOR}

A careful design of the LGS wavefront sensor is crucial for the performance of the LTAO system. Currently all LGS WFSs are using a Shack-Hartmann sensor, other sensors, like the pyramid, ${ }^{7}$ have been suggested but not yet used routinely on an existing facility. With the SH-WFS, the challenge comes from the elongation of the source images in the focal plane on the detector (Fig. 8). This elongations results from the paraxial projection of the section of Na layer crossed by the Na Laser. For the GMT telescope with the side launch configuration, this elongation is 6" for a $10 \mathrm{~km}$ thick Na layer with the telescope pointing at zenith. The thickness of the Na layer varies with time ${ }^{9}$ and can reach up to $15 \mathrm{~km}$ i.e. 9.6" at zenith. The elongation decreases with the telescope elevation, so does the Na photon return (Fig. 9) due to the increase distance to the Na layer.

The elongation of the WFS spots may lead to error in the centroids due to ${ }^{1,10}$ (i) the finite field-of-view of the WFS subaperture which truncates the image of the Na layer (Fig. 10, left image) and (ii) the under-sampling of the elongated spots due to the finite resolution of the detector (Fig. 10, center image). For example, for a $15 \mathrm{~km}$ thick $\mathrm{Na}$ layer and Nyquist sampling the diffraction limited image of a $50 \mathrm{~cm}$ aperture requires 39 pixels per spot. For a $50 \times 50$ lenslet array, this would require a $2 \mathrm{k}$ pixels square array.

Hopefully, the sampling can be reduced noting that the typical size of a non elongated LGS image is usually not the diffraction limit but the results of the uplink and downlink propagation of the Laser through the atmosphere. 


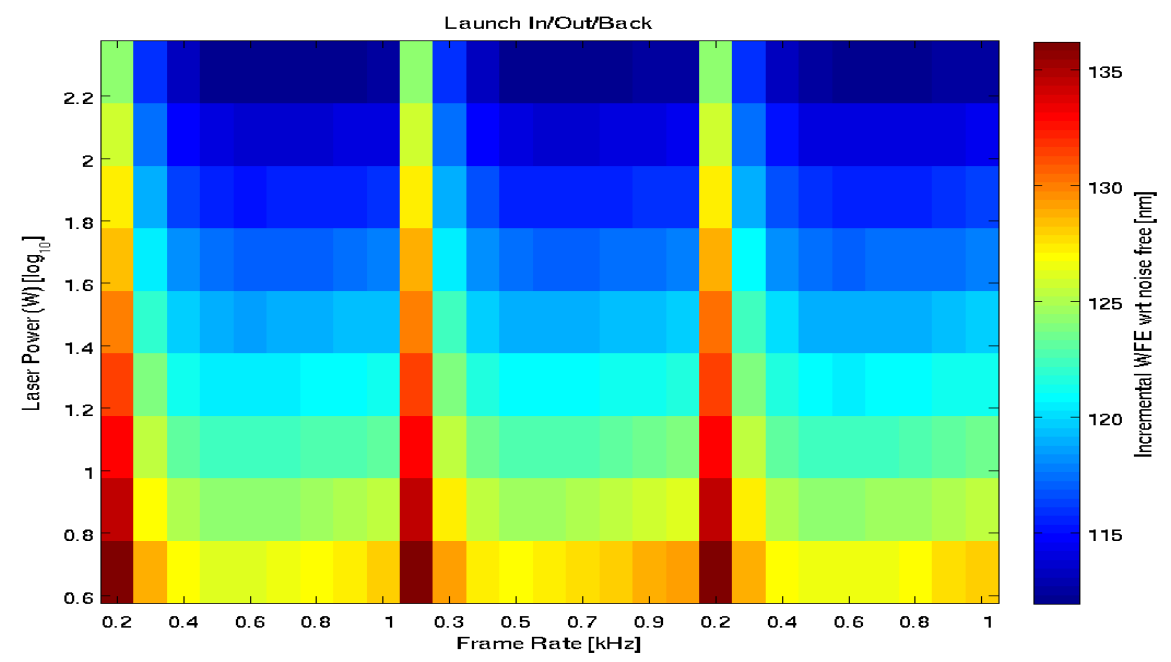

Figure 6. LGS tomography WFE versus LGS WFS detector frame rate and Laser power for different LGS launch location. From left to right, the maps correspond to 3 different launch telescope locations, within the primary segments, from the telescope edge and behind the ASM.

The typical spot size is around 1". Nyquist sampling a spot of that size leads to 19 pixels required to sample a $15 \mathrm{~km}$ thick Na layer.

Considering that the Na thickness is less than $13 \mathrm{~km} 90 \%$ [moussaoui] of the time i.e. 8.3 " at zenith, a $12 \times 12$ centroid pixel window with 0.71 " per pixel has been selected for the sampling of the elongated spots. With 1 guard pixel on each side, this leads to $14 \times 14$ pixels per subaperture. This has been found to be the proper trade-off to mitigate errors induced by low sampling and small field-of-view.

Fig. 11 plots the LTAO system WFE as a function of the lenslet array size from $40 \times 40$ to $70 \times 70$. The blue curve plots the quadratic sum of the fitting and the tomographic error derived from analytic expressions. The green curve plots the WFE derived from the end-to-end model in the case of non-elongated spot and without noise. This WFE includes fitting, tomographic and temporal errors. The red curve corresponds to the elongated spots case for the same simulations. The LGS aberration are now part of the WFE in addition to the former errors. It is worth noting the minimum around $60 \times 60$. The increasing WFE for smaller lenslet array is supposed to be due to the increasing turbulence aberration per subaperture as the subaperture size is increasing with respect to $r_{0}$. On the other hand, there is also an increase of the WFE for larger lenslet array, this can be due either to numerical errors in the inversion of the covariance matrices as they grow with the 4th power of the lenslet arrays or to the increase of tip-tilt power per subaperture as the lenslet size decreases. The cyan curve adds photon noise and read-out noise to the elongated spots. The read-out noise is set to 3 photo-detected event per pixel rms and the number of photon is set to $349 \mathrm{ph} / \mathrm{cm}^{2} / \mathrm{s}$ corresponding to the lowest expected Na return in a year. Here too a minimum at $60 \times 60$ is found.

Consequently the baseline opto-mechanical design of the LGS WFS assembly (Fig. 12 and Fig. 13) includes a SH-WFS with a $60 \times 60$ lenslet array. The detector resolution must be $60 \times 14=840$ pixels with a read-out of at least 500fps. The proposed detector is the NGSD developed by E2V embedded inside a camera package designed by First Light Imaging.

The mechanical design incorporates a translating stage to accommodate the Na layer range from $82.5 \mathrm{~km}$ to $200 \mathrm{~km}$ and a rotating stage to compensate the rotation of the LGS induced by the rotating gregorian platform supporting the instrument with the LTAO system.

Thee diameter and focal distance of the launch telescopes are of importance to the size of the Laser spot on the Na layer and on the LGS WFS detector. A $30 \mathrm{~cm}$ diameter launch telescope with an adjustable focal length around a nominal value of $23 \mathrm{~km}$ has been selected. 


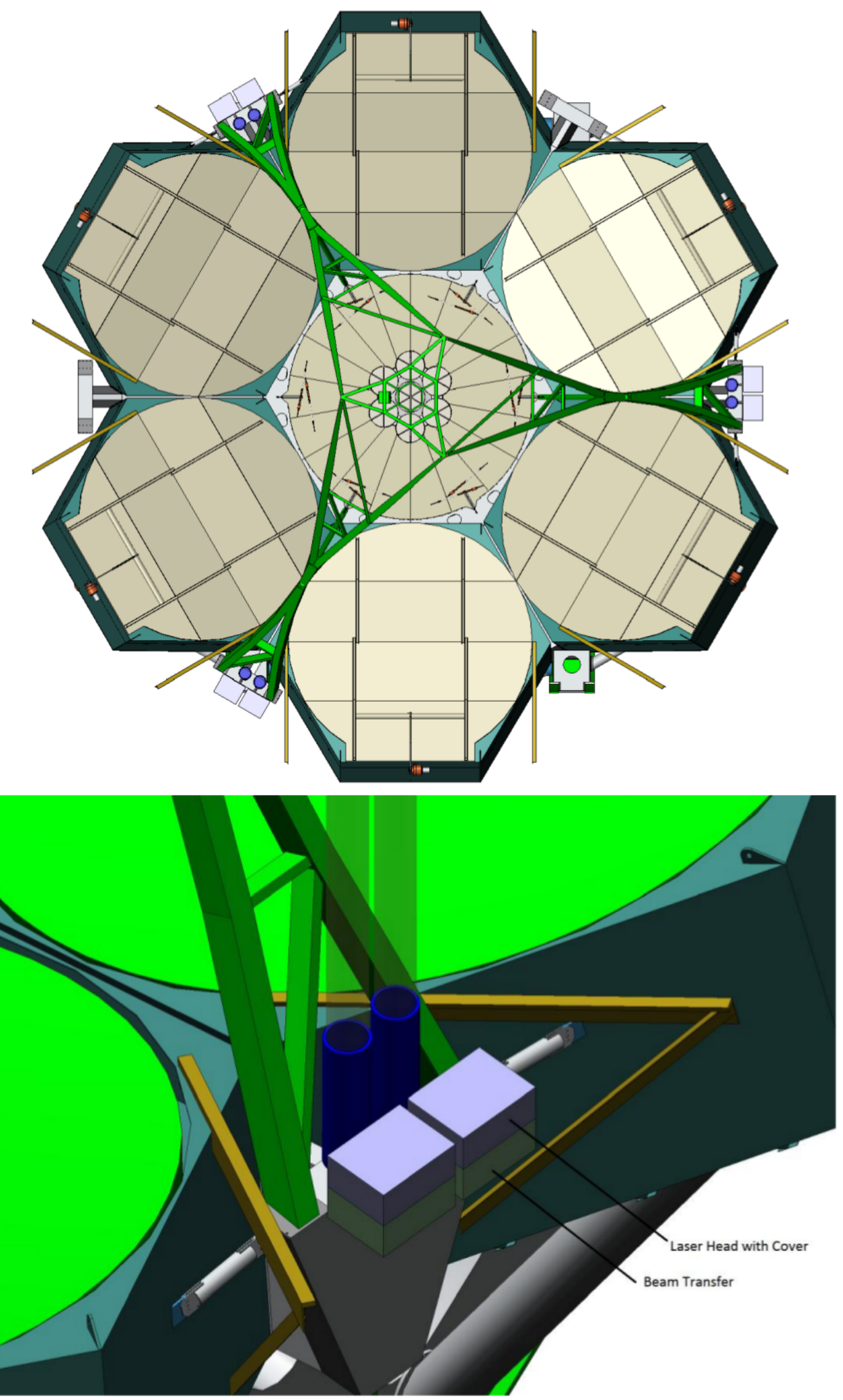

Figure 7. Laser launch telescope locations with respect to the primary mirror cells. 


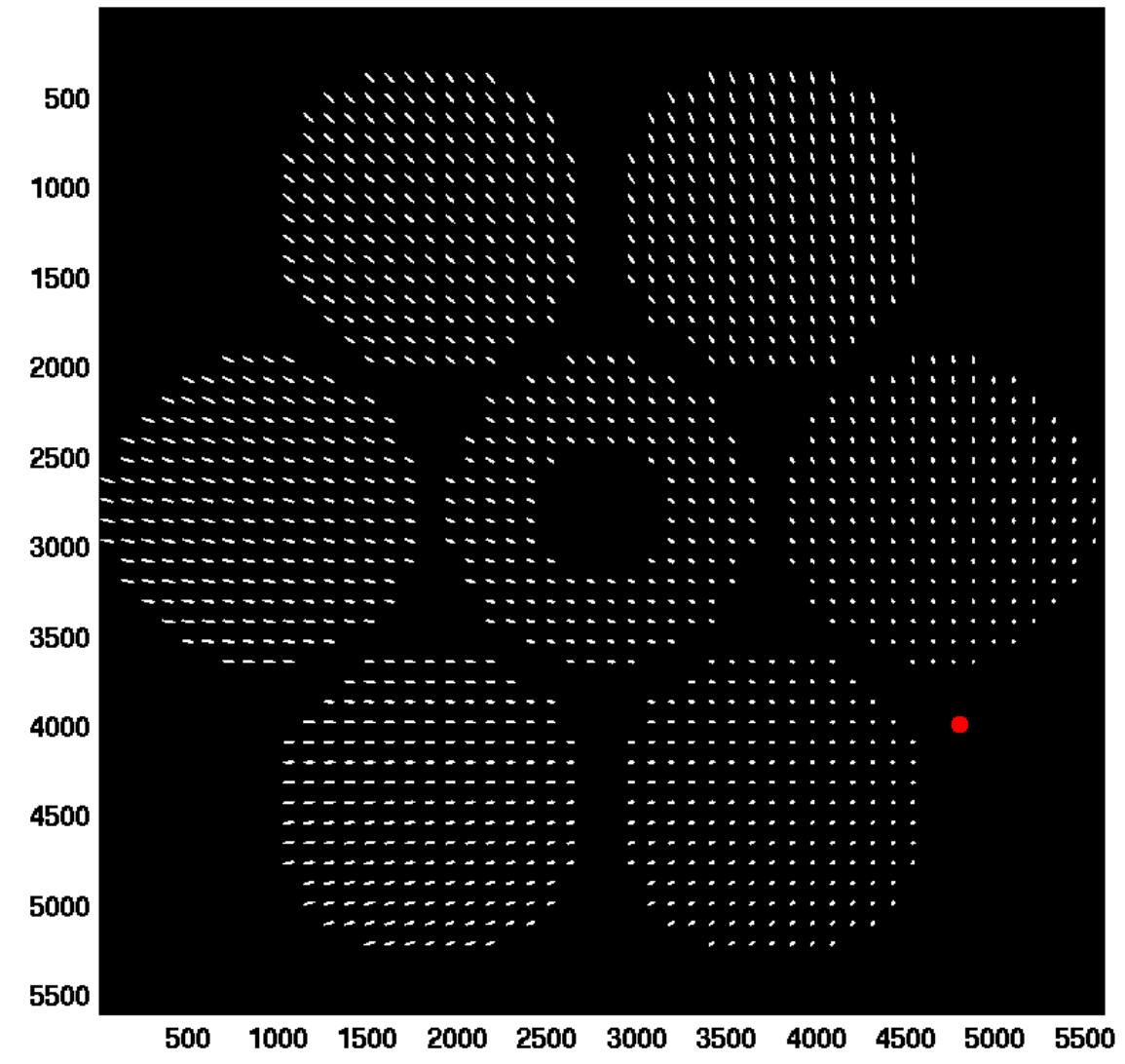

Figure 8. LGS WFS detector frame for a $50 \times 50$ lenslet array. The pixel scale is 0.71 " and the subaperture field-of-view is $7.1 "$. The red dot shows the location of the launch telescope. 


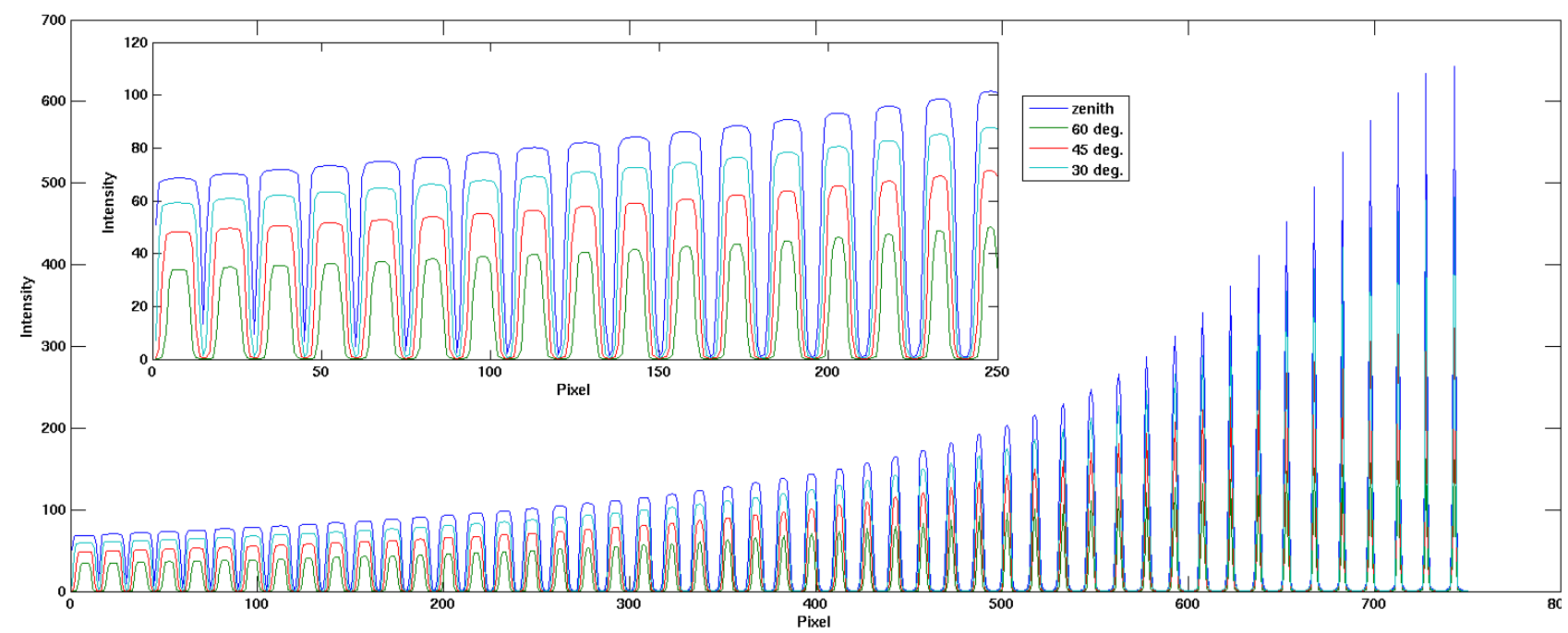

Figure 9. Cut of a LGS WFS detector frame along a line going through the telescope axis and thought the launch telescope. A full $25 \mathrm{~m}$ pupil is used in this simulation. The Na layer is $10 \mathrm{~km}$ thick with a top-hat profile. The detector frame has been computed for different zenith angle showing that both the spot elongation and the spot intensity decrease when the distance to the $\mathrm{Na}$ layer increases.
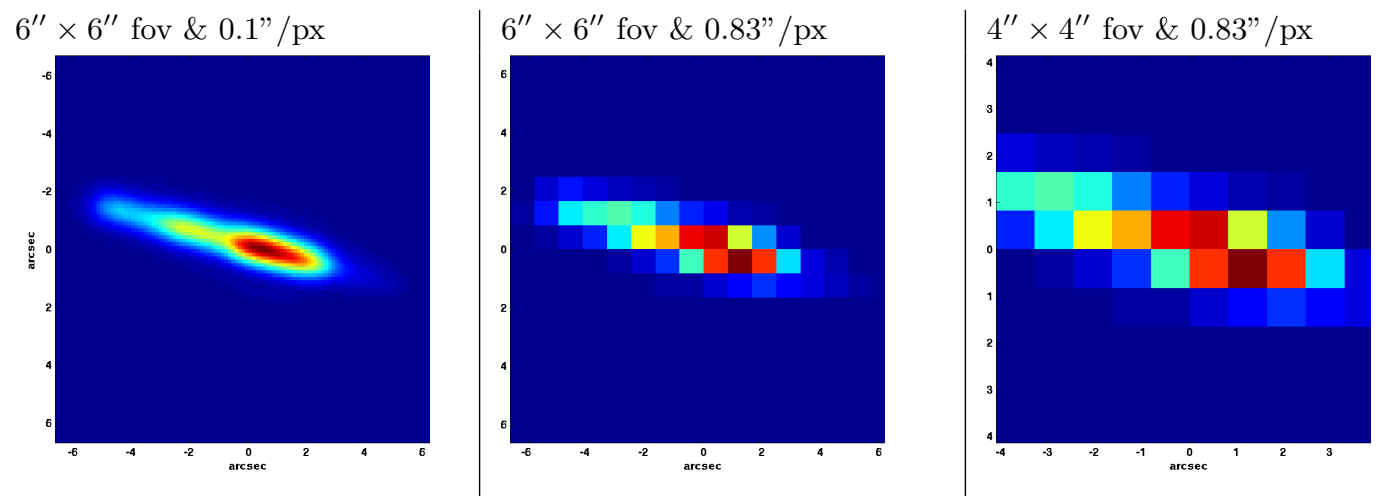

Figure 10. The left image is an example of an elongated spot with a large field-of-view (12") and a fine sampling (0.1"/px). The effect of a coarser sampling is showed in the middle and the effect of a reduced field-of-view is showed in the right image. 


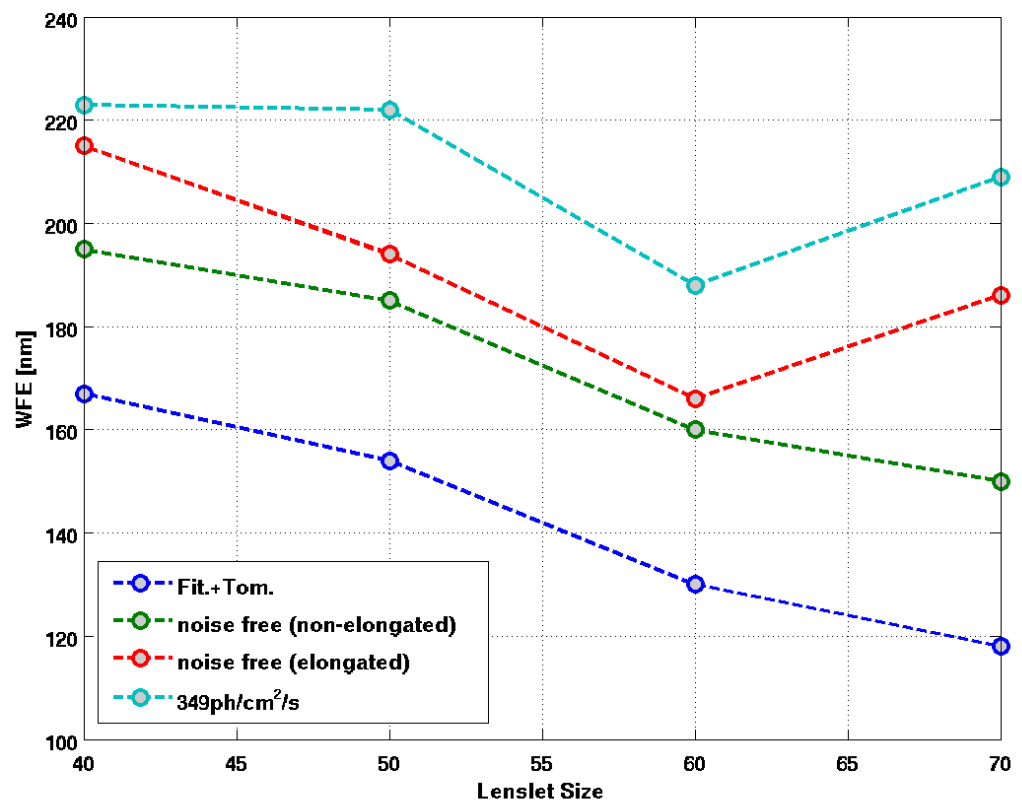

Figure 11. End-to-end simulations of the GMT LTAO system: the WFE is plotted as a function of the size of the lenslet array of the LGS WFS.

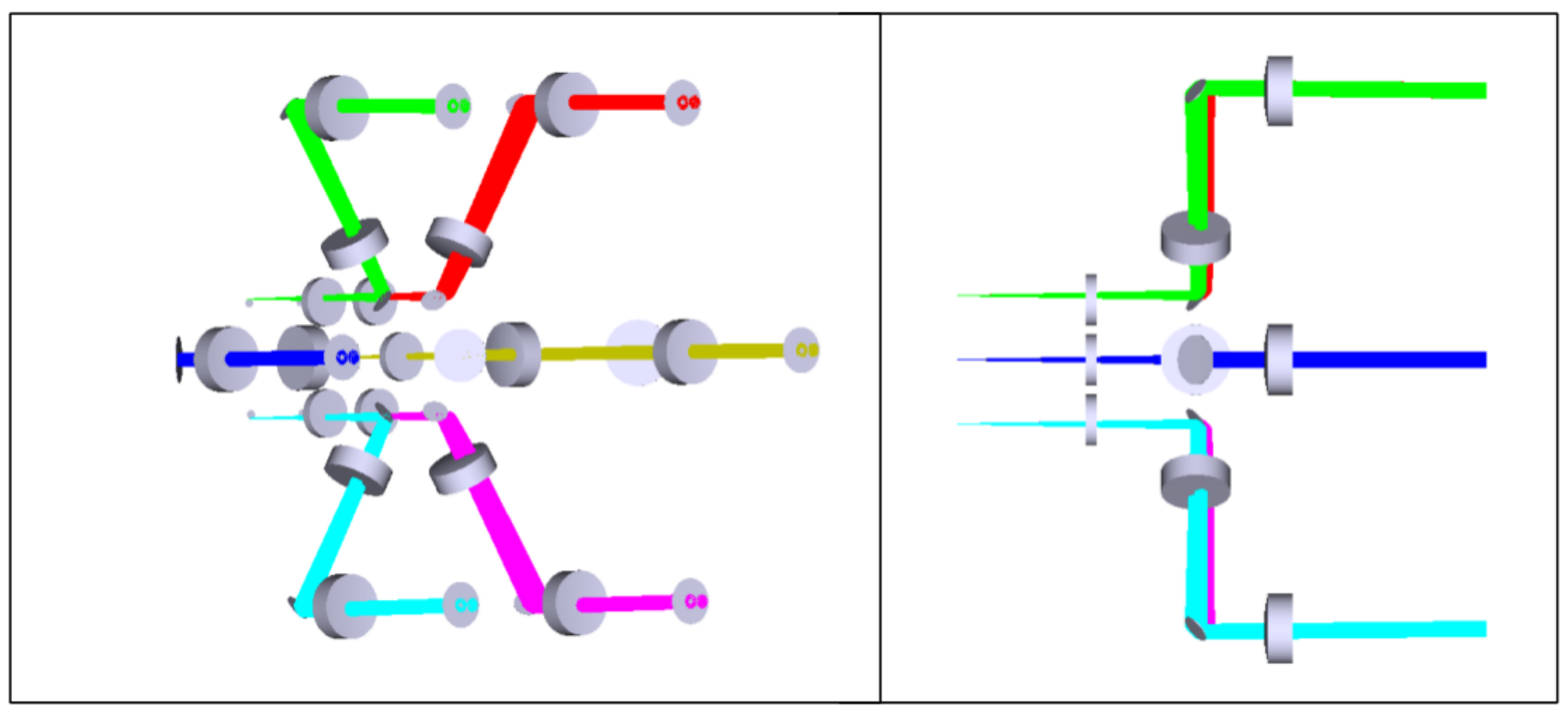

Figure 12. Optical layout of the 6-channel assembly of the LGS WFSs from the telescope focal plane after the tertiary mirror to the lenslet array of the SH-WFS. 


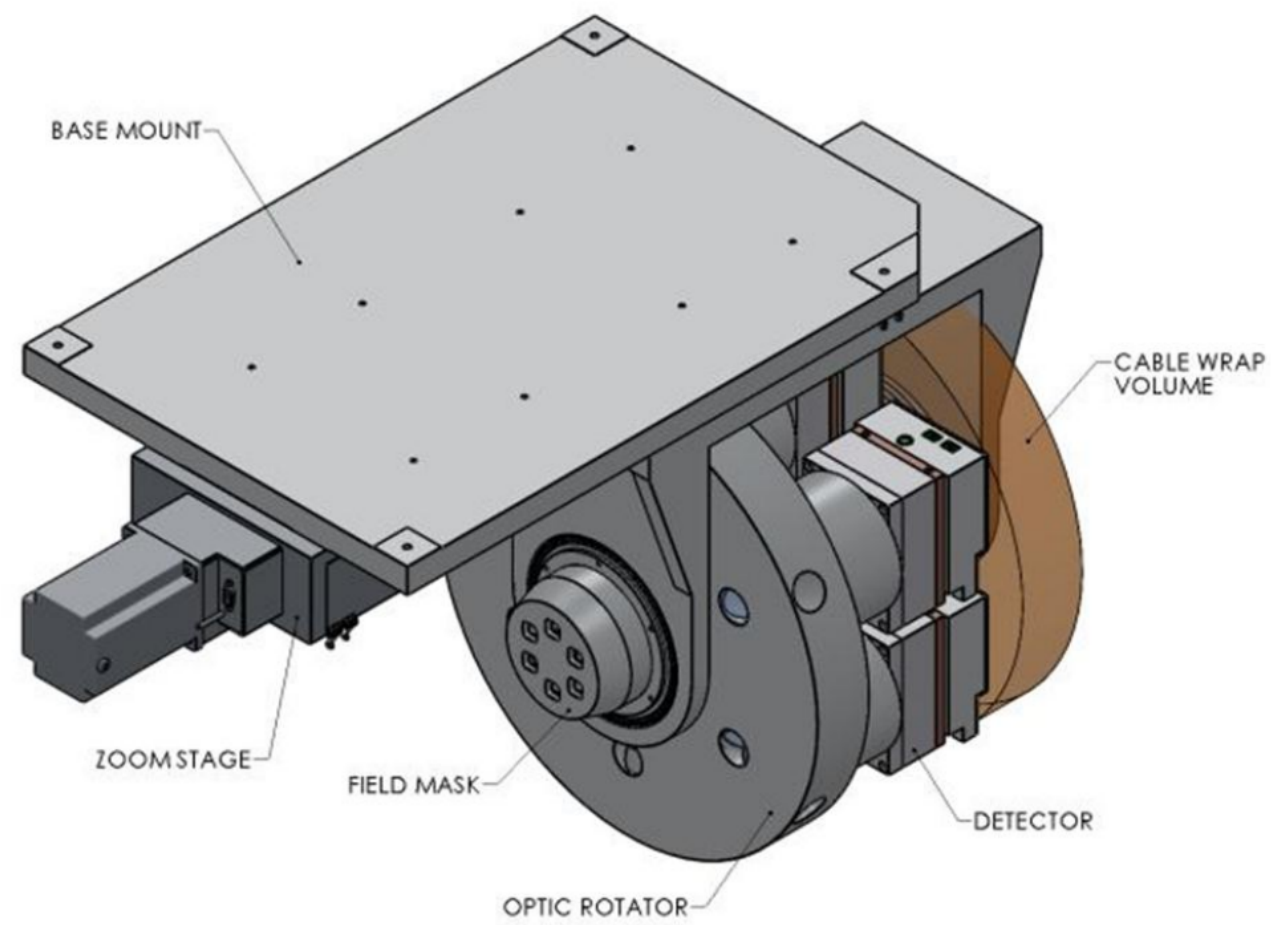

Figure 13. Mechanical assembly of the LGS WFSs of the GMT LTAO system.

\section{THE ON-INSTRUMENT WAVEFRONT SENSOR}

\subsection{Tip-tilt sensing}

The LGS wavefront sensor cannot measure the tip-tilt ${ }^{11}$ due to the round-trip through the atmosphere of first the Laser and then the Na fluorescence. A NGS must be found within the 90" field-of-view of the instrument to estimate the tip-tilt. The tip-tilt performance of the system will be then limited by the anisokinetism error which reaches up to 13 mas at 1.5 arcmin off-axis (Fig. 14).

Fig. 15 draws isopleths of TT residual jitter in mas using either $3 \mathrm{R}$-band TT star (left graph) or $1 \mathrm{~K}$-band AO corrected TT star (right graph). It clearly shows that a single AO corrected TT star in $\mathrm{K}$-band is preferable to any combination of NGS visible TT sensors. Within the 90" field-of-view of the instrument, it is obvious that finding 1 suitable TT star is easier that finding 3 of them. Fig. 16 plots the residual TT jitter for 1 AO-corrected $\mathrm{K}$-band TT star as a function of the probability of encountering such star within a 90" circle at the galactic pole. The solid lines and the square markers correspond to analytic and end-to-end simulation respectively. In blue, only the ASM is used and in red, both the ASM and a DM in the TT path are used. It shows that the gain of adding another DM occurs for TT star more than 40" away from the telescope axis.

\subsection{Focus sensing}

In the $\mathrm{Na}$ layer, the vertical distribution of $\mathrm{Na}$ atom density is varying with time. This creates time fluctuations in the photo-center of Na layer which induces a focus error in the LGS WFSs. A dedicated focus sensor is then needed to track those variations. Fig. 17 plots the rms WFE due to the fluctuations in the mean altitude of the Na layer. In order to keep the WFE below 30nm, the focus sensor need to operate at about $10 \mathrm{~Hz}$ or higher.

\subsection{Truth sensing and segment piston sensing}

The science instrument needs to host 2 more sensors. A truth sensor is needed for calibration of static aberrations and tracking of quasi-static aberrations. A piston sensor is also required to maintain the segment phasing accuracy at better than $50 \mathrm{~nm}$ rms. Details on these sensors are given in Ref. ${ }^{13}$ 


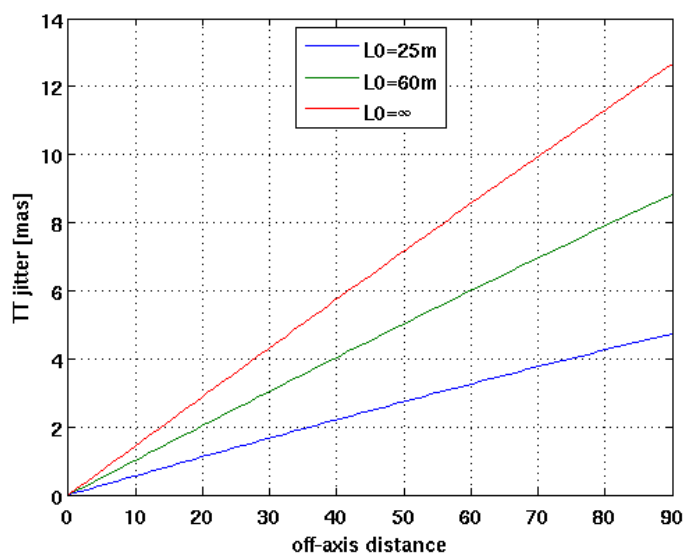

Figure 14. Anisokinetism error as a function of the off-axis distance of the TT star for different values of the out scale $\mathcal{L}_{0}$.
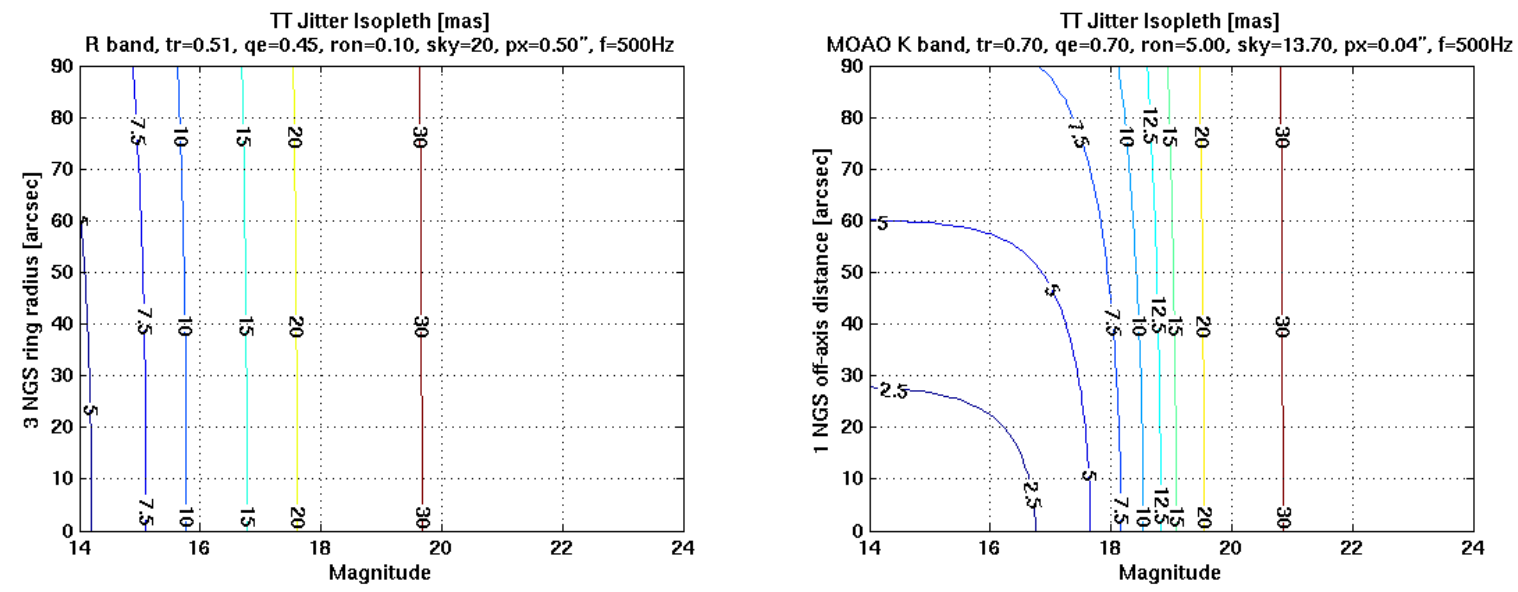

Figure 15. Isopleths of residual tip-tilt atmospheric jitter using either with 3 R-band NGSs (left graph) or with 1 K-band AO corrected NGS (right graph). 


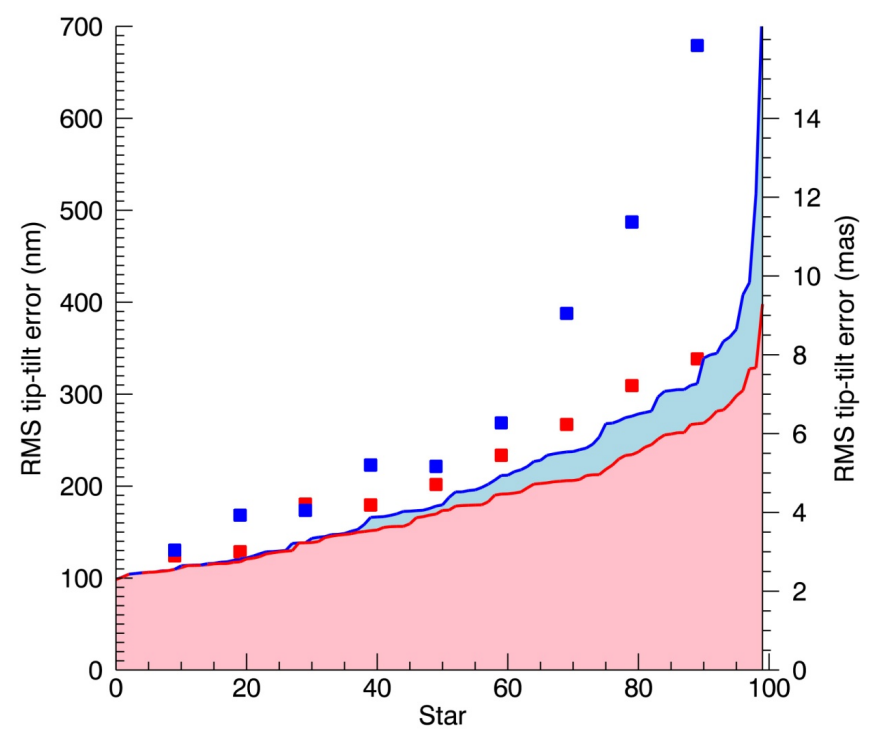

Figure 16. Probability to find $1 \mathrm{~K}$-band TT star: analytic (solid lines) versus end-to-end (square markers). In blue, only the ASM is used in the simulation and in red both the ASM and a DM in the TT path are used.

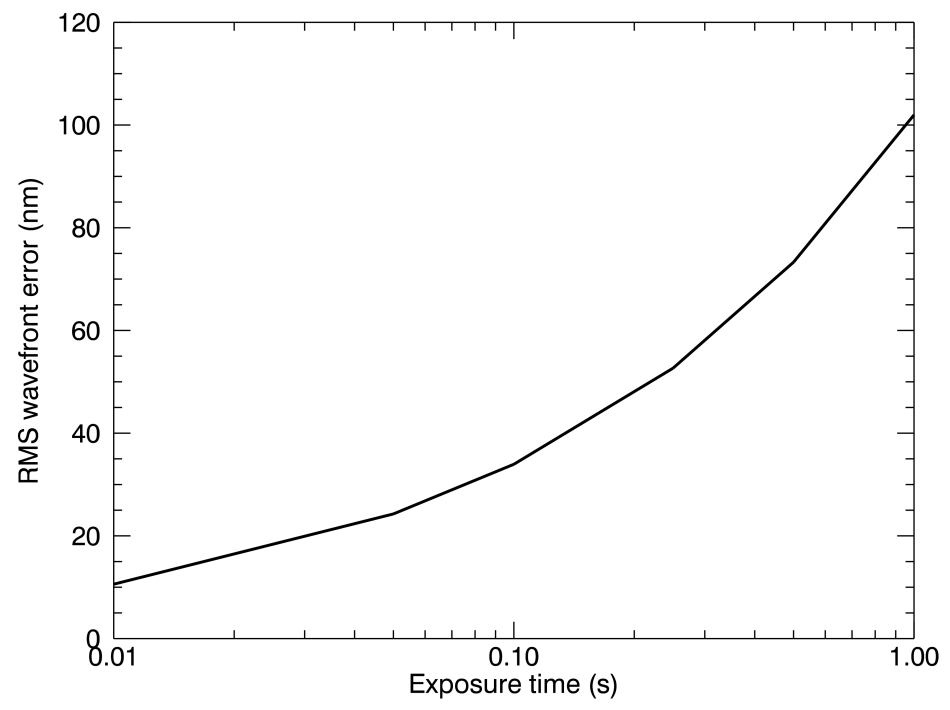

Figure 17. RMS wavefront error due to $\mathrm{Na}$ altitude residual fluctuations 


\section{CONCLUSION}

The preliminary design of the GMT Laser Tomography Adaptive Optics System is currently developed collaboratively between the GMT Organization project office and the Australian National University. The Preliminary Design includes four subsystems required for the Laser Tomography Adaptive Optics:

1. The LTAO Wavefront Sensor Subsystem,

2. The On-Instrument WFS,

3. The Laser Guide Star Facility ,

4. The Wavefront Processor.

Trade Studies and detailed analyses have been conducted to determine the optimum architecture and wavefront sensing techniques within the constraints of the existing telescope design. Based on the analysis conducted, the following decisions have been made for the LTAO system:

- The wavefront will be corrected by a seven-segment adaptive secondary mirror with 672 actuators each.

- There will be a constellation of six sodium laser guide stars (LGS) in a regular hexagon with a diameter of $60 "$ centered on the science target.

- Six laser launch telescopes will each launch a 20-25 Watt laser guide star. The launch telescopes will be located between the primary mirrors at a radius of approximately $11.5 \mathrm{~m}$ from the telescope optical axis.

- Six Shack-Hartmann wavefront sensors with $60 x 60$ subapertures across the pupil will be used to measure the wavefront in the direction of the laser guide stars. Each subaperture will have 14x14 pixels with a pixel scale of 0.71 per pixel.

- The wavefront will be reconstructed tomographically using a minimum mean-squared-error reconstructor operating in pseudo open-loop.

- Tip-tilt will be measured using an on-instrument infrared detector operating light from the $\mathrm{K}$ band and reading a quad cell at $500 \mathrm{~Hz}$ to $1 \mathrm{kHz}$. The anisoplanatism in the direction of the tip-tilt sensor will be partially compensated with a 32x32 MEMS DM.

- Image motion from wind induced vibration will be sensed in the science instrument using the tip-tilt sensor.

- Truth, focus and segment piston will be measured with the J- to H-bands light of the tip-tilt star.

The above design choices result in a design solution for the LTAO Mode of the GMT AO System that will produce less than $250 \mathrm{~nm}$ RMS of high order wavefront error and less than 6 mas of image motion over $50 \%$ of the Sky.

\section{Acknowledgments}

"This work has been supported by the GMTO Corporation, a non-profit organization operated on behalf of an international consortium of universities and institutions: Astronomy Australia Ltd, the Australian National University, the Carnegie Institution for Science, Harvard University, the Korea Astronomy and Space Science Institute, the Smithsonian Institution, The University of Texas at Austin, Texas A\&M University, University of Arizona and University of Chicago. This material is based in part upon work supported by AURA through the National Science Foundation under Scientific Program Order No. 10 as issued for support of the Giant Segmented Mirror Telescope for the United States Astronomical Community, in accordance with Proposal No. AST-0443999 submitted by AURA." 


\section{REFERENCES}

1. Richard M. Clare, Marcos A. van Dam, and Antonin H. Bouchez. Modeling low order aberrations in laser guide star adaptive optics systems. Opt. Express, 15(8):4711-4725, 2007.

2. B. L. Ellerbroek and C. R. Vogel. Simulations of closed-loop wavefront reconstruction for multiconjugate adaptive optics on giant telescopes. In R. K. Tyson \& M. Lloyd-Hart, editor, Society of Photo-Optical Instrumentation Engineers (SPIE) Conference Series, volume 5169 of Society of Photo-Optical Instrumentation Engineers (SPIE) Conference Series, pages 206-217, December 2003.

3. Antonin H. Bouchez et al. The Giant Magellan Telescope adaptive optics program. Number 8447-54 in Proc. SPIE, 2012.

4. Philip M. Hinz, Guido Brusa-Zappellini, Vidhya Vaitheeswaran, Thomas McMahon, Thomas Connors, Russell P. Knox, Manny Montoya, and Antonin H. Bouchez. Design and predicted performance of the GMT ground-layer adaptive optics mode. Number 8447-137 in Proc. SPIE, 2012.

5. Matt Johns, Patrick J. McCarthy, Keith Raybould, Antonin Bouchez, Arash Farahani, Jose M. Filgueira, George H. Jacoby, Stephen A. Shectman, and Michael Sheehan. Giant Magellan Telescope: overview. Number 8444-52 in Proc. SPIE, 2012.

6. S.M. Kay. Fundamentals of Statistical Signal Processing: Estimation Theory. Prentice Hall, New-Jersey, 2010.

7. B. Le Roux. Pyramid wavefront sensing with a laser guide star for an ELT. In Society of Photo-Optical Instrumentation Engineers (SPIE) Conference Series, volume 7736 of Society of Photo-Optical Instrumentation Engineers (SPIE) Conference Series, July 2010.

8. Peter J. McGregor, Gabe J. Bloxham, Robert Boz, John Davies, Matthew C. Doolan, Michael Ellis, John Hart, Jon J. Nielsen, Simon Parcell, Robert G. Sharp, and Dejan Stevanovic. The GMT integral-field spectrograph (GMTIFS) conceptual design. Number 8446-53 in Proc. SPIE, 2012.

9. N. Moussaoui, B. R. Clemesha, R. Holzlöhner, D. M. Simonich, D. Bonaccini Calia, W. Hackenberg, and P. P. Batista. Statistics of the sodium layer parameters at low geographic latitude and its impact on adaptive-optics sodium laser guide star characteristics. Astron. Astrophys., 511:A31, February 2010.

10. O. Lardière and R. Conan and C. Bradley and K. Jackson and P. Hampton. Characterization and mitigation of laser-guide-star aberrations. In Adaptive Optics for Extremely Large Telescopes, June 2009.

11. F. Rigaut and E. Gendron. Laser guide star in adaptive optics : The tilt determination problem. Astron. Astrophys., 261:677-684, 1992.

12. Gelys Trancho, Brady Espeland, Antonin H. Bouchez, Rodolphe Conan, Philip M. Hinz, and Marcos A. van Dam. GMT AO system requirements and error budgets in the preliminary design phase. Number 8447-202 in Proc. SPIE, 2012.

13. Marcos A. van Dam, Rodolphe Conan, Antonin H. Bouchez, and Brady Espeland. Design of a truth sensor for the GMT laser tomography adaptive optics system. Number 8447-43 in Proc. SPIE, 2012. 